\title{
Artificial intelligence in safety system
}

\author{
Li-Shan Chen ${ }^{1}$, Yen-Ming Tseng ${ }^{2,}$, and Xiao-Na Lin ${ }^{1}$ \\ ${ }^{1}$ School of Management, Fujian University of Technology, No.3 Xueyuan Road, Fuzhou University \\ Town, 350108, Fuzhou City, Fujian Province, China \\ ${ }^{2}$ College of Intelligence Robot, Fuzhou Polytechnic, No.8 Lianrong Road, Fuzhou University Town, \\ 350108, Fuzhou City, Fujian Province, China
}

\begin{abstract}
This research aims to study learning environment, and let the learning environment become smart. Swarm intelligence, cloud computing, and active Ultra-High Frequency RFID were used on it. We built friendly human-computer-interface software for users to use as pad phone. The Extensible Markup Language (XML) and C sharp language were used in this research. If the users begin to search, the kernel safety learning system automatically communicates with other RFID readers by agents, and the agents can search the closer camera for users. This study's result has successfully implemented to Chin-Huo educational organization, and it would be helpful for the paterfamilias to hold all situations about their children at Chin-Huo educational organization. Paterfamilias can understand their children's learning, going to Chin-Huo and leaving ChinHuo through personal computers, or notebooks simultaneously or asynchronously by the computer-mediated communication. That will be great help in the grip of whole after-school remedial education, teaching and learning situation.
\end{abstract}

\section{Introduction}

In many applications, the environmental context for and drivers of movement patterns are just as important as the patterns themselves [1]. Kopf et al. [2] use a standard technique to estimate scene depth, but assign depths to image gradients rather than pixels. Digital artifacts created via transformational technologies often embody implicit knowledge that must be correctly interpreted to successfully act upon the artifacts [3]. With continued advances in communication network technology and sensing technology, there is astounding growth in the amount of data produced and made available through cyberspace.

Sun and Chen [4] [5] apply adopts the Windows Media Player along the RTP/RTSP protocol in order to embed the mobile information system into the users' pad phone, and provides a solution (including hardware solutions) to promote campus safety environment. Chen combines the swarm intelligence and Web Services to transform a conventional library system into an intelligent library system having high integrity, usability, correctness, and reliability software for readers [6]. L. S. Chen, and S. L. Chen [7] built the intelligent system and developed a knowledge base of the computer-parts.

*Corresponding author: swk1200@qq.com 
This research used the swarm intelligence, cloud computing, and active Ultra-High Frequency RFID for learning environment, and built friendly human computer interface software for users to use as pad phone. We program the system and software with Extensible Markup Language (XML) and C sharp language.

\section{Literature review}

\subsection{For cloud computing}

Hayes et al. [8] show how leveraging an underlying platform-as-a-service (PaaS) cloud model can provide integration with web service patient monitoring systems while providing high availability, scalability, and security. The emergence of cloud computing has led enterprises to rethink fundamentally how they organize their content assets. In particular, IT managers are challenged to decide what content should be managed in the cloud and what should not [9]. Lian, Yen and Wang [10] investigate the critical factors that will affect the decision to adopt cloud computing technology in developing countries, specifically in Taiwan's hospital industry. Ranjan and Zhao [11] advance the management and delivery of services in large, heterogeneous, uncertain, and evolving cloud computing environments.

\subsection{For swarm intelligence}

The explosive growth of mobile apps has given rise to the challenge of finding out interesting apps for users. Recommender systems are employed to meet this challenge. However, as the lack of user and app data, the development of recommender systems for mobile apps is still at a slow pace [12]. The multi-level thresholding is one of the most important issues in image segmentation. It is a time consuming problem, i.e. finding appropriate threshold values could take exceptionally long computational time [13]. Swarm robotics is an approach to collective robotics that takes inspiration from the self-organized behaviors of social animals. Through simple rules and local interactions, swarm robotics aims at designing robust, scalable, and flexible collective behaviors for the coordination of large numbers of robots [14].

\subsection{For RFID}

Broekmeulen, and Donselaar [15] suggest a replenishment policy for perishable products which takes into account the age of inventories and which requires only very simple calculations. Zhou [16] takes a different perspective by modeling item-level information visibility in general. Delgado, Ros, and Vila [17] present a system that is able to process the information provided by a Tagged World to identify user's behavior and to produce alarms in dangerous situations. Abad, Palacio, Nuin, Zárate, Juarros, Gómez, and Marco [18] present important advantages regarding conventional traceability tools and currently used temperature data loggers such as more memory, reusability, no human participation, no tag visibility needed for reading, possibility of reading many tags at the same time and more resistance to humidity and environmental conditions. Lee, and Chan [19] propose a genetic algorithm to determine such locations in order to maximize the coverage of customers. Also, the use of RFID is suggested to count the quantities of collected items in collection points and send the signal to the central return center. 


\section{Research methods}

This research used the swarm intelligence, cloud computing, and active Ultra-High Frequency RFID for learning environment, and built friendly human computer interface software for users to use as pad phone. The Extensible Markup Language (XML) and C sharp language were used in this research. The framework of RFID system was shown as Figure 1, and its searching process was shown as Figure 2. In Figure 1, there were eight RFID Readers and eight IP-Cameras for the safety learning system. In Figure 2, the agents generator would make agents which could search the optimum path of RFID Reader.

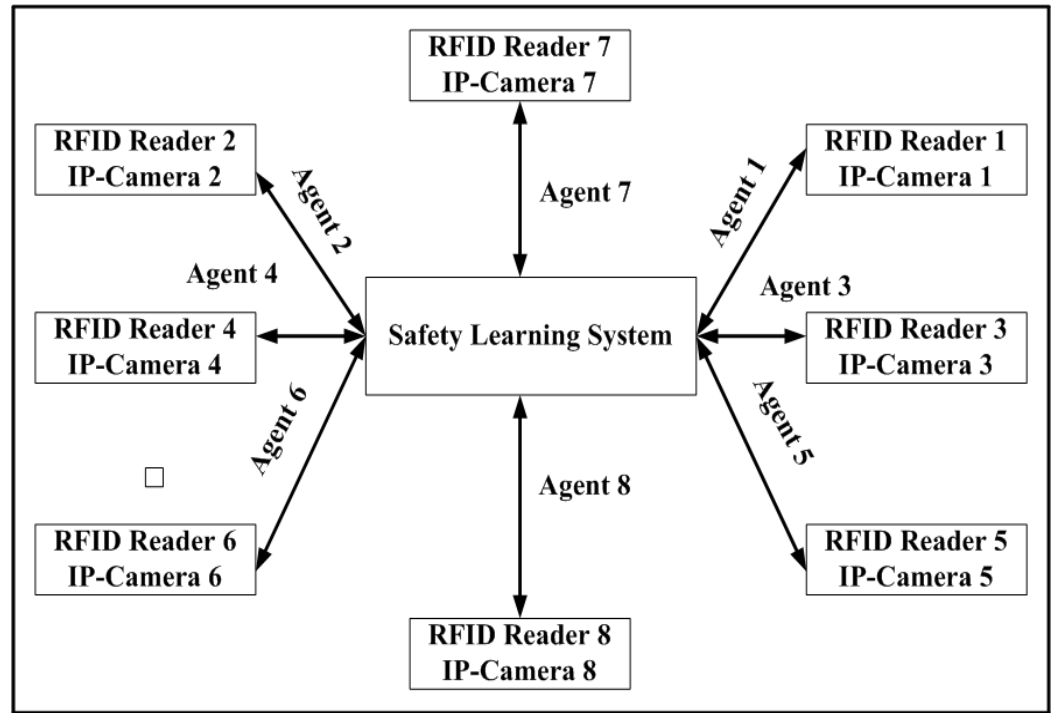

Fig. 1. Framework of RFID system.

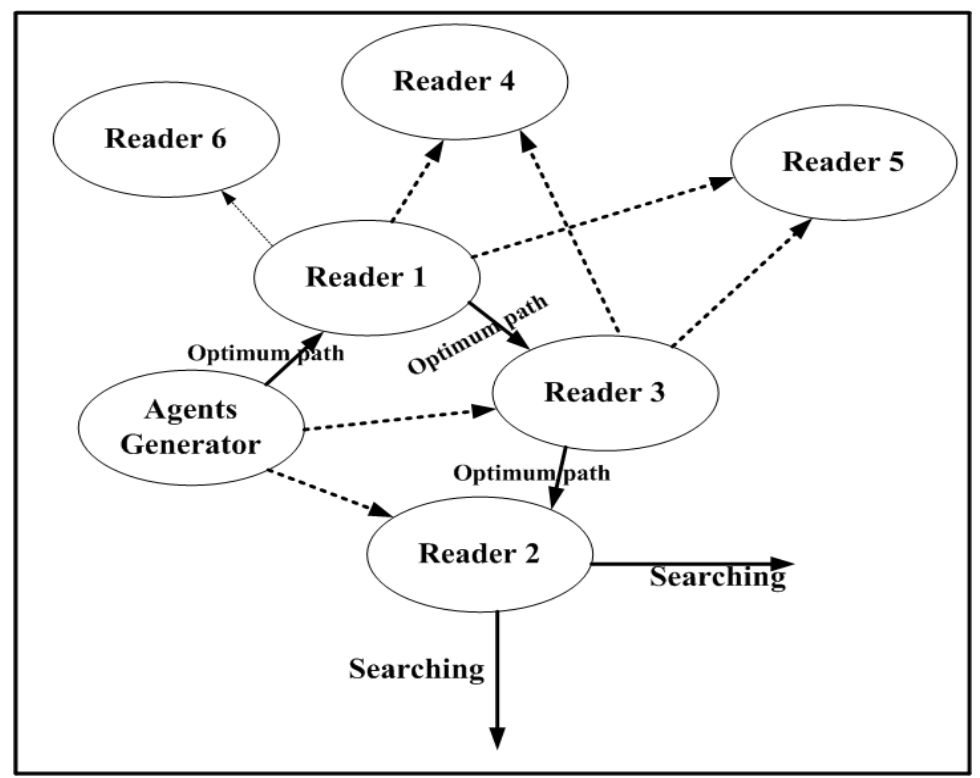

Fig. 2. Searching process. 


\section{Research results}

The safety system has been successfully developed, as shown in Figures 3-6. Figure 3 is the login frame. Figure 4 is welcome frame. There are eight areas in this safety system; (1) The left side of the gate, (2) The right side of the gate, (3) The eastern side of the house, (4) House Back, (5) Classroom 1, (6) Classroom 2, (7) Off ice, (8) Leisure area, as shown in Figure 5. Figure 6 is the searching results of classroom 1.

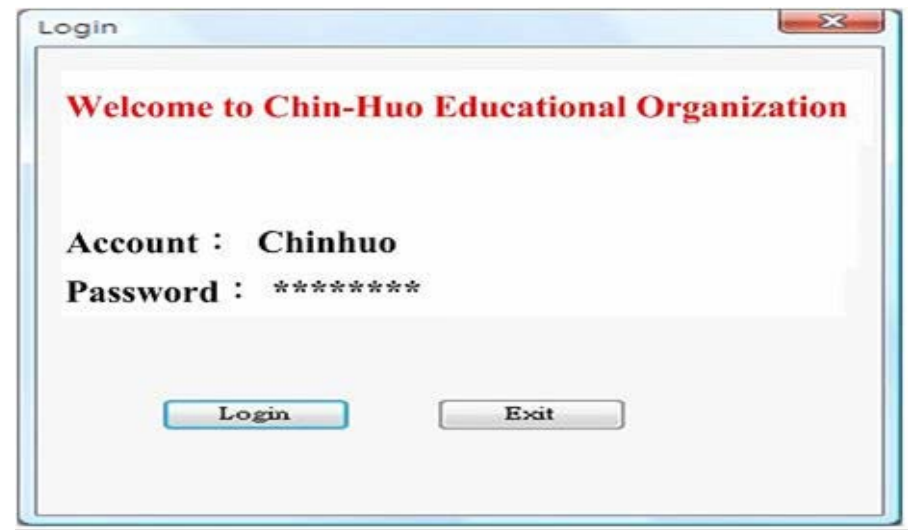

Fig. 3. Login frame.

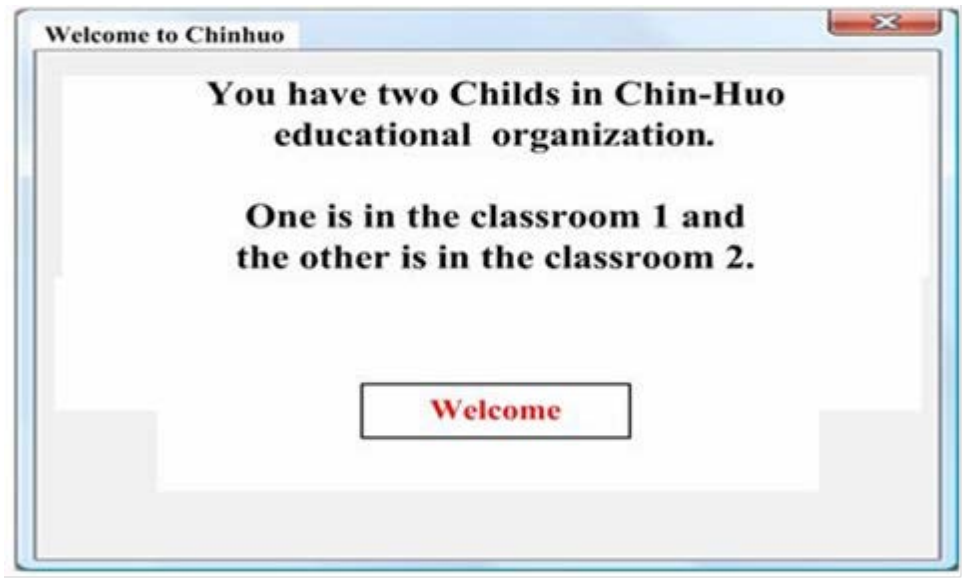

Fig. 4. Welcome frame. 


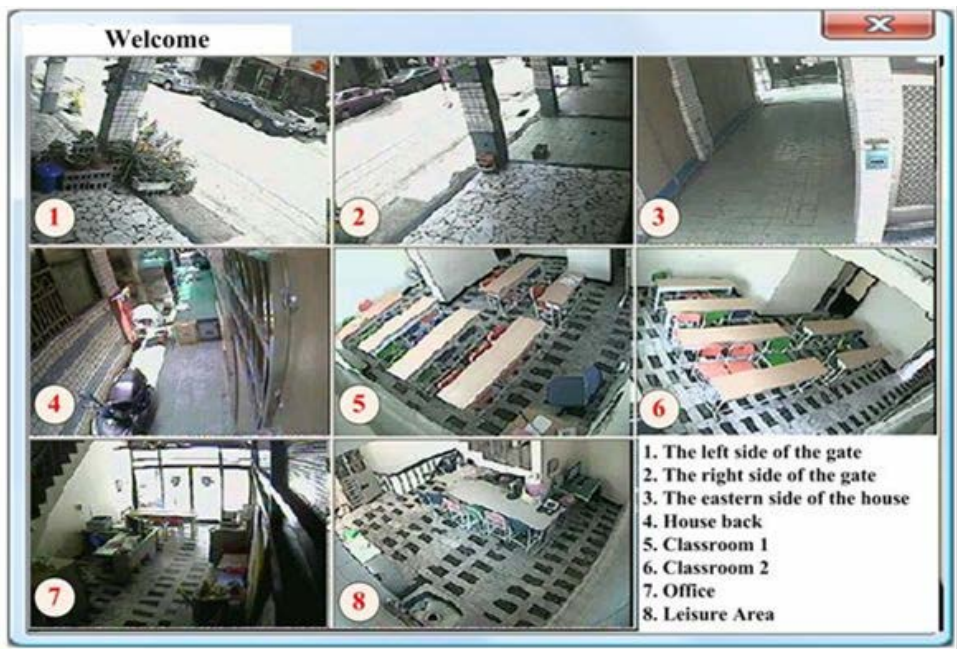

Fig. 5. Eight areas.

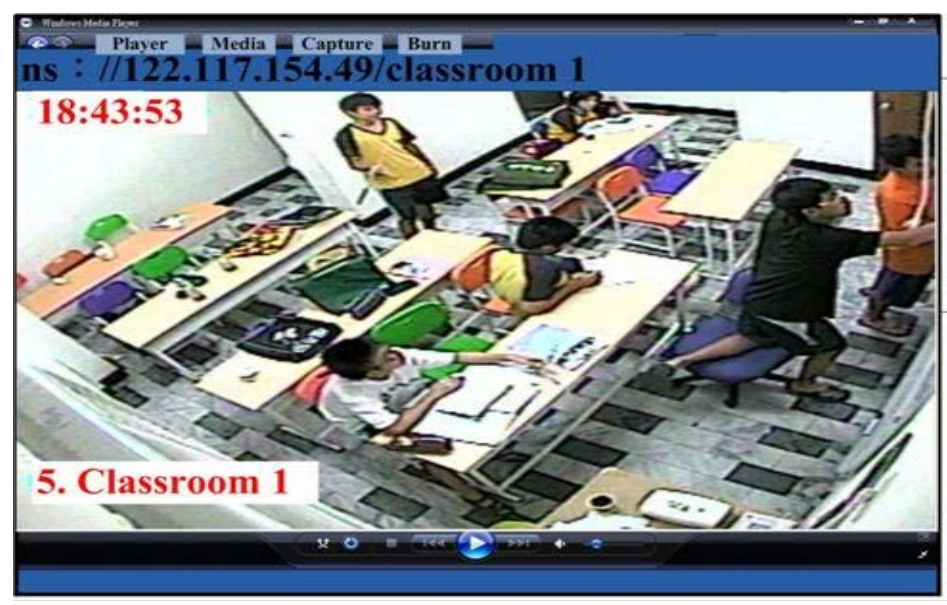

Fig. 6. Searching results (Classroom 1).

\section{Conclusions}

This research aims to study learning environment, and let the learning environment become smart. Swarm intelligence, cloud computing, and active Ultra-High Frequency RFID were used on it. We built friendly human-computer-interface software for users to use as pad phone. The Extensible Markup Language (XML) and C sharp language were used in this research. The paterfamilias' time in operating the instrument was saved. Even someone who does not have the professional knowledge. This research also develops friendly human computer interface software for users to use as personal computers or notebooks. The size of the software is 30 kilobits; therefore, the software is not a liability for the users' tools. The result has been successfully implemented on Chin-Huo educational organization and it would be helpful for the paterfamilias to hold all situations about their 
children at one educational organization. It will be of great help in the grip of whole afterschool remedial education, teaching and learning.

\section{References}

1. S. Bleischa, M. Duckhama, A. Galtonb, P. Laubec, J. Lyond. IJGIS, Vol. 28, 363-382 (2014)

2. J. kopf, F. Langguth, D. Scharstein, R. Szeliski, M. Goesele. ACM, TOG Homepage archive, Vol. 32, No. 199(2013)

3. P.M. Leonardi, D. E. Bailey, MIS Quarterly, 32 (2), 411-436 (2011)

4. S.Y. Sun, L.S. Chen, IJOI, Vol. 6, 63-67 (2014)

5. S.Y. Sun, S.Y.,L. S. Chen, IJOI, Vol. 6, 167-178 (2013)

6. L.S. Chen. LCATS, Vol.34, 1-10 (2010)

7. L.S. Chen. LCATS, Vol.32, 127-141 (2009)

8. G. Hayes, K. El-Khatib, C. McGrego, CLIEE, Vol. 260,1149-1158 (2014)

9. I. Gonzenbach, C. Russ, J.V. Brocke, ECMISRP,145-161 (2014)

10. J.W. Lian, D.C. Yen, Y.T. Wang, IJIM, Vol. 34, 28-36 (2014)

11. R. Ranjan, L. Zhao, JS, Vol. 65, 154-184 (2013)

12. X. Xia, X. Wang, X. Zhou, T. Zhu, LNIEE, Vol. 274, 405-412 (2014)

13. A. Marciniak, M. Kowal, P. Filipczuk, J. Korbicz, ISTMDAISC, Vol. 230, 301-311 (2014)

14. M. Brambilla, E. Ferrante, M. Birattari, M. Dorigo, SI, Vol. 7, 1-41(2013)

15. R. A.C.M. Broekmeulen, K. H. V. Donselaar, COR, 36 (11), 3013-3018(2015).

16. W. Zhou, EJOR, 198 (1), 252-258 (2009)

17. M. Delgado, M. Ros, M. A. Vila, ESWA, 36 (6), 9899-9906 (2015)

18. E. Abad, F. Palacio, M. Nuin, A. G. Zárate, A. Juarros, J. M. Gómez, S. Marco, JFE, 93 (4), 394-399 (2014)

19. C.K. M, T. M. Lee, T.M. Chan, ESWA, 36 (5), 9299-9307 (2015) 